\title{
Phase transitions, interfacial fluctuations and hidden symmetries for fluids near structured walls
}

\author{
A O PARRY ${ }^{1}$ and J M ROMERO-ENRIQUE ${ }^{1,2}$ \\ ${ }^{1}$ Department of Mathematics, Imperial College, 180 Queen's Gate, London SW7 2BZ, UK \\ ${ }^{2}$ Departamento de Física Atómica, Molecular y Nuclear, Area de Física Teórica, \\ Universidad de Sevilla, Apartado de Correos 1065, 41080 Sevilla, Spain \\ E-mail: a.o.parry@ic.ac.uk
}

\begin{abstract}
Fluids adsorbed at micro-patterned and geometrically structured substrates can exhibit novel phase transitions and interfacial fluctuation effects distinct from those characteristic of wetting at planar, homogeneous walls. We review recent theoretical progress in this area paying particular attention to filling transitions pertinent to fluid adsorption near wedges, which have highlighted a deep connection between geometrical and contact angles. We show that filling transitions are not only characterized by large scale interfacial fluctuations leading to universal critical singularities but also reveal hidden symmetries with short-ranged critical wetting transitions and properties of dimensional reduction. We propose a non-local interfacial model which fulfills all these properties and throws light on long-standing problems regarding the order of the 3D short-range critical wetting transition.
\end{abstract}

Keywords. Wetting; filling; effective Hamiltonians; covariance; non-locality.

PACS Nos 68.08.Bc; 05.70.Np; 68.35.Rh; 05.10.Cc

\section{Introduction}

The last decade has seen some highly impressive technological advances which allow the controlled fabrication and tailoring of micropatterned and structured solid surfaces on the nanometre to micrometre scale [1], and that are crucially important to the emerging microfluidic industry [2]. From a more fundamental point of view, the influence of the surface character can be rather dramatic and radically alter the character of the fluid adsorption compared to that ocurring at planar, homogeneous walls [3-5]. A simple example of how the substrate geometry can influence the fluid adsorption is provided by a simple wedge geometry [6-14]. This paper reviews recent advances in the understanding of fluid wedge adsorption, focussing on the mesoscopic scale where we anticipate the interfacial fluctuations may play an important role.

In this paper, we will first revisit the filling transition, which refers to the phase transition from microscopic to macroscopic adsorption as $\theta \rightarrow \alpha^{+}$, where $\pi-2 \alpha$ is 
the wedge opening angle and $\theta$ is the sessile drop contact angle. Previous studies showed that the filling transitions is continuous under less restrictive conditions than for continuous wetting at planar walls $[10,11]$ and present far stronger largescale fluctuations than those characteristic of critical wetting transitions reflecting the anisotropy of soft-mode interfacial fluctuations induced by the wedge geometry. On the other hand, two-dimensional wedge filling in the fluctuation-dominated regime in both ordered and disordered systems show scaling properties which are identical with short-ranged critical wetting transitions. The only influence of the wedge geometry is to shift the effective value of the contact angle from $\theta$ to $\theta-\alpha-\mathrm{a}$ feature which has been referred to as wedge covariance [14]. This 'hidden symmetry' between wetting and filling appears to restrict the allowed values of the critical exponents at both $2 \mathrm{D}$ filling and wetting and leads to some new insights into the properties of critical wetting transitions.

Here we show that, in addition to this fluctuation-induced wedge covariance, there is a classical wedge covariance for 3D systems with short-ranged forces which is supported by microscopic Landau-like calculations for both shallow and acute wedges. Thus classical covariance provides a stringent test for any interfacial model to be physically acceptable.

We argue that the general theory of short-ranged wetting at structured walls should be formulated using a non-local interfacial model. This is somewhat analogous to important advances in density functional models [15]. The interactions in the interfacial model have a natural physical interpretation in terms of bulklike correlations arising from tube-like fluctuations [16] between the unbinding interface and the wall which contribute towards a binding-potential functional $W[l, \psi]$. In the limit of small interfacial fluctuations the NL model identically recovers the known form of the local Hamiltonian, including the position-dependence term which arises from the theory of critical wetting proposed by Fisher and Jin (FJ) [17], allowing one to trace the specific position dependence of the binding potential and position-dependent stiffness to the Ornstein-Zernike (OZ) bulk correlation function. When applied to the wedge filling with arbitrary tilt angles the model obeys the classical wedge covariance relations. Finally, this model may throw some light on the problem of the order of the 3D short-range critical wetting of planar interfaces. Renormalization group (RG) analysis and computer simulations show the non-perturbative influence of NL interactions. Despite precise connection with the FJ model at a perturbative level, our results show that the full NL model has no stiffness instability which may drive the transition first-order (as in the FJ model [18]) and the wetting transition remains second order. The size of the asymptotic critical regime for various observables is also discussed.

\section{Background}

Consider a wedge formed from the intersection of two smooth, planar walls that meet at angles $\alpha$ to the $z=0$ plane (see figure 1), so that its opening angle is $\pi-2 \alpha$. We represent the parallel displacement vector in the $z=0$ plane as 


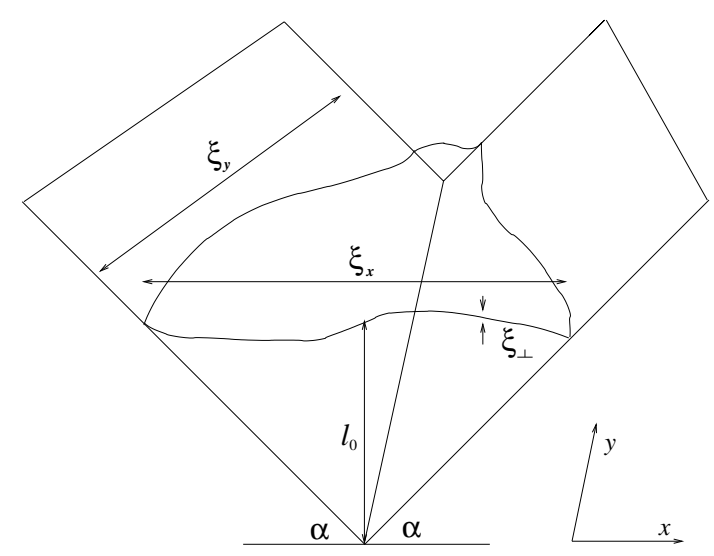

Figure 1. Schematic illustration of a typical interfacial configuration in the $3 \mathrm{D}$ wedge geometry and the typical diverging length scales at the filling transition. Note that $l_{\mathrm{w}}=\left\langle l_{0}\right\rangle$.

$\mathbf{x}=\left(x, y_{1}, \ldots, y_{D-2}\right)$ where the Cartesian coordinates $x$ and $y_{1}, \ldots, y_{D-2}$ measure the distances across and along the wedge, respectively, and $D$ is the dimensionality of the system. The height of the wall above the $z=0$ plane is described by a height function $\psi(\mathbf{x})=\tan \alpha|x|$. The wedge is in contact with a bulk vapour at subcritical temperature $T$ (for later convenience, we will consider $k_{\mathrm{B}} T=1$, defining the energy scale), and chemical potential at saturation conditions $\mu=\mu_{\text {sat }}(T)^{-}$. The wedge preferentially adsorbs liquid near its bottom provided the contact angle $\theta(T)$ of the sessile drop (defined for the planar wall-fluid interface) is less than $\pi / 2$. Under these assumptions, a phase transition between a partial and complete filling (i.e. between situations in which the wedge adsorption is microscopic and macroscopic, respectively) was predicted by Concus and Finn [6] (see also [7] and $[8]$ ). We sketch here their argument for the $3 \mathrm{D}$ case. The total grand potential $\Omega$ contains the following contributions:

$$
\Omega=-p V+\sigma_{\mathrm{wv}} A+f_{\mathrm{w}} L,
$$

where the pressure $p$, the surface tension $\sigma_{\mathrm{wv}}$ of the wall-vapour interface and the excess wedge free energy $f_{\mathrm{w}}$ are the conjugated fields to the accessible volume of the fluid $V$, the wedge area $A$ and the length $L$ along the wedge. The excess wedge free energy has a thermodynamic contribution which arises from the fact that the wedge is filled to a height $l_{\mathrm{w}}$ where the liquid-vapour interface is macroscopically flat. This contribution can be written as

$$
f_{\mathrm{w}}=\frac{2 \Sigma(\cos \alpha-\cos \theta) l_{\mathrm{w}}}{\sin \alpha},
$$

where $\Sigma$ is the liquid-vapour surface tension (stiffness for anisotropic systems) and we have used Young's equation. It is clear from eq. (2) that for $\theta<\alpha$ the free energy can be lowered by completely filling the wedge (i.e. $l_{\mathrm{w}} \equiv \infty$ ), while for $\theta>\alpha$ the equilibrium value of $l_{\mathrm{w}}$ is finite and arises from a balance between 
thermodynamics and internal energy (intermolecular forces) or entropy (fluctuations) contributions. So, the condition for the wedge filling transition is given by the elegant expression:

$$
\theta\left(T_{\mathrm{f}}\right)=\alpha,
$$

where $T_{\mathrm{f}}$ is the filling transition temperature. This prediction remains unchanged when interfacial fluctuations are also considered. Note that this condition is fulfilled for a temperature smaller than the wetting transition value $T_{\mathrm{w}}$ (assuming the usual scenario where the contact angle decreases with the temperature). Filling transitions may be first or second order corresponding to an infinite jump or a continuous divergence of the mean interfacial height $l_{\mathrm{w}}$ respectively. The conditions for continuous filling are less restrictive than for continuous wetting and wedges made from walls that exhibit first-order wetting may still exhibit continuous filling $[10,11]$.

As a critical phenomenon, the critical filling transition is characterized by a set of critical exponents which describe the divergence of the relevant length scales (see figure 1): the average mid-point height $l_{\mathrm{w}}$, the perpendicular correlation length $\xi_{\perp}$ at the mid-point, and the correlation lengths $\xi_{x}$ and $\xi_{y}$ describing fluctuations across and along the wedge, respectively. Obviously, for the $2 \mathrm{D}$ case $\xi_{y}$ does not exist. If we define the temperature-like scaling field $t \propto\left(T_{\mathrm{f}}-T\right) \propto(\theta-\alpha)$, at bulk coexistence these length scales diverge as

$$
l_{\mathrm{w}} \sim t^{-\beta_{\mathrm{w}}}, \quad \xi_{\perp} \sim t^{-\nu_{\perp}}, \quad \xi_{y} \sim t^{-\nu_{y}}
$$

while $\xi_{x}$ is trivially related to the mean height via $\xi_{x} \sim l_{\mathrm{w}} \cot \alpha$.

We are interested in the effect the capillary wave-like interfacial fluctuations can have on the critical behaviour of the second-order filling transitions. Consequently, we consider effective Hamiltonian models which describe the fluctuations of a collective coordinate $l(\mathbf{x})$ representing the local height of the interface. This coarse-grained description is valid at length scales much bigger than the bulk correlation length of the adsorbed phase. For planar substrates, the simplest version corresponds to the capillary-wave $(\mathrm{CW})$ model:

$$
H_{\pi}[l]=\int \mathrm{d} \mathbf{x}\left\{\frac{\Sigma}{2}(\nabla l)^{2}+W(l)\right\},
$$

where $\Sigma$ is the stiffness coefficient of the unbinding interface which can be identified as the surface tension for isotropic fluids, and $W(l)$ is the binding potential. However, their generalization to the wedge geometry (and more complex geometries) is far from obvious. A very reasonable, and for many purposes satisfactory, model is the drumhead-like interfacial model proposed by Rejmer, Dietrich and Napiórkowski (RDN) [9]:

$$
H_{\mathrm{RDN}}=\int \mathrm{d} \mathbf{x}\left\{\Sigma\left[\sqrt{1+(\nabla l)^{2}}-1\right]+\sec \alpha W(\cos \alpha[l-\psi(\mathbf{x}])\}\right.
$$

where $l(\mathbf{x})$ denotes the vertical height relative to the $\mathbf{x}=0$ plane and $\psi(\mathbf{x})=$ $\tan \alpha|x|$. Note that the square gradient term of the CW model has been replaced 
by the correct rotationally invariant expression for the total area of the liquidvapour interface. This ensures that the model recovers the correct filling phase boundary. Secondly, the interface interacts with the closest substrate via the corresponding planar binding potential where the distance is taken to be normal to the substrate. These assumptions are certainly valid far from the wedge bottom, where the interface and wall are parallel. However, it can be seen that the interface is blind to the wedge bottom since points for distances $|x|<l_{\mathrm{w}} \sin \alpha \cos \alpha$ do not contribute to the binding potential.

For small $\alpha$, the RDN model simplifies to [9]:

$$
H[l]=\int \mathrm{d} \mathbf{x}\left\{\frac{\Sigma}{2}(\nabla l)^{2}+W(l-\alpha|x|)\right\} .
$$

The binding potential $W(l)$ decays at large distances as $W(l) \sim-a l^{-p}$, where the Hamaker constant $a$ is positive in the temperature region of interest whilst the value of exponent $p$ depends on the specific range of the forces with $p=2,3$ for non-retarded and retarded van der Waals forces, respectively. It is also possible to modify the model to include the effect of quenched disorder but for the moment we concentrate on pure systems with thermal fluctuations.

We first consider the 3D case. Minimization of the capillary-wave-like model (7) determines the following mean-field values of the critical exponents for $3 \mathrm{D}$ filling $[10]$

$$
\beta_{\mathrm{w}}=\frac{1}{p}, \quad \nu_{\perp}=\frac{1}{4}, \quad \nu_{y}=\frac{1}{2}+\frac{1}{p} .
$$

However, fluctuation effects will modify these values, since fluctuations are extremely anisotropic at wedge filling with $\xi_{y} \gg \xi_{x}$ and are dominated by pseudoone-dimensional local translations in the height of the filled region along the wedge $[10,11]$ (the 'breather-mode' excitations). Tilt and torsional modes can also be included, but they do not affect the critical behaviour [19]. Mean-field exponents will be valid only for intermolecular potentials with $p<4$ for which $\xi_{\perp} \ll l_{\mathrm{w}}$. The filling fluctuation (FFL) regime corresponds to potentials with $p>4$ and a pseudoone-dimensional wedge Hamiltonian which accounts only for the breather-mode excitations can be used to study the filling transition $[10,11]$ :

$$
H_{\mathrm{w}}\left[l_{0}\right]=\int \mathrm{d} y\left\{\frac{\Sigma l_{0}}{\alpha}\left(\frac{\mathrm{d} l_{0}}{\mathrm{~d} y}\right)^{2}+V\left(l_{0}\right)\right\},
$$

where $l_{0}(y) \equiv l(0, y)>0$ is the local height of the interface above the wedge bottom and $l_{\mathrm{w}}=\left\langle l_{0}(y)\right\rangle$. The model is considered valid only for small wavevectors $k_{y} \ll k_{y}^{\max } \sim \xi_{x}^{-1}$. However, because the fluctuations at filling are strongly anisotropic the relevant scaling combination $\xi_{y} k_{y}^{\max }$ diverges in the scaling limit and the cut-off does not determine universal quantities. The most important feature of this effective model is the presence of a bending term resisting fluctuations along the wedge which is proportional to the local interfacial height. At bulk coexistence the wedge potential $V\left(l_{0}\right)$ has the form

$$
V\left(l_{0}\right)=\frac{\Sigma}{\alpha}\left(\theta^{2}-\alpha^{2}\right) l_{0}+C l_{0}^{1-p}
$$


and for $\theta>\alpha$ has a minimum located at the mean-field value of $l_{\mathrm{w}}$. Note that the first term in the wedge binding potential is the small $\alpha$ approximation to the thermodynamic term $f_{\mathrm{w}}$ (eq. (2)) and is proportional to the linear, temperature-like scaling field $t$.

The bending term in eq. (9) is invariant under the renormalization group rescaling $y \rightarrow y^{\prime}=y / b, l_{0} \rightarrow l_{0}^{\prime}=l_{0} / b^{\zeta_{\mathrm{w}}}$, with $\zeta_{\mathrm{w}}=1 / 3[10,11]$. Under this transformation the linear scaling field $t$ and wedge Hamaker constant $C$ rescale to $t^{\prime}$ and $C^{\prime}$ with

$$
t^{\prime}=b^{4 / 3} t, \quad C^{\prime}=b^{(4-p) / 3} C .
$$

This result shows that the temperature-like scaling field is always relevant and that the intermolecular forces are relevant and irrelevant for $p<4$ and $p>4$ respectively. These results can also be obtained from mean-field theory if we assume that the effective wedge potential has a fluctuation contribution $D l_{0}^{-\tau_{\mathrm{w}}}$, where $D \propto \Sigma$ and $\tau_{\mathrm{w}}=2 / \zeta_{\mathrm{w}}-3[11]$. The values of the exponents in the FFL regime are

$$
\beta_{\mathrm{w}}=\frac{1}{4}, \quad \nu_{\perp}=\frac{1}{4}, \quad \nu_{y}=\frac{3}{4} .
$$

Recent computer simulation studies of the Ising model are in agreement with these predictions $[20,21]$. From eqs (8) and (12) it is clear that fluctuations are enhanced by geometry, since the interfacial roughness is much larger than that corresponding to a wetting situation in a planar substrate. This fact can be understood from the effective dimensional reduction for the relevant fluctuations [19].

The previous results can be generalized to a $D$-dimensional wedge which is translationally invariant in the $D-2$ dimensions along the wedge $[10,11]$ and in the presence of random-bond disorder [19]. Breather-mode excitations do not lead to large-scale interfacial roughness for $D>D_{\text {c }}$, where $D_{\text {c }}$ is the upper critical dimension, and mean-field theory is valid. The upper critical dimension satisfies the relation $\zeta\left(D_{\mathrm{c}}-1\right)=0$, where $\zeta(D)$ is the interfacial wandering exponent of a $D$-dimensional planar interface [19]. For $D<D_{\text {c }}$ we find two possible fluctuation regimes corresponding to mean-field-like and fluctuation-dominated behaviour. The FFL occurs for sufficiently short-ranged potentials with $p>2\left(1 / \zeta_{\mathrm{w}}-1\right)$ with a generalized wedge wandering exponent $\zeta_{\mathrm{w}}$ and critical exponent for the divergence of the interfacial height $\beta_{\mathrm{w}}$ given in terms of $\zeta(D-1)$ and $\zeta(D)$ as [19]

$$
\zeta_{\mathrm{w}}=\frac{\zeta(D-1)}{1+\zeta(D-1)-\zeta(D)}, \quad \beta_{\mathrm{w}}=\frac{\zeta(D-1)}{2(1-\zeta(D))} .
$$

Intriguingly, the $\beta_{\mathrm{w}}$ exponent for pure systems (thermal disorder) has the correct two-dimensional limit $\beta_{\mathrm{w}}=1$ known from explicit transfer-matrix results for contact potentials $[12,13]$. In general, the $2 \mathrm{D}$ breather-mode picture can be explicitly obtained from transfer-matrix analysis and renormalization group calculations $[22]$.

The $2 \mathrm{D}$ critical wedge filling presents an unexpected connection with the critical wetting transition on a planar substrate [12-14]. Although both transitions are clearly different, explicit calculations for thermal and random-bond systems show that at the FFL regime the probability distribution function (PDF) of the midpoint wedge interfacial height $P_{\mathrm{w}}(l ; \theta, \alpha)$ can be written in terms of the interfacial 
height $\mathrm{PDF} P_{\pi}(l ; \theta)$ corresponding to a critical wetting transition at the strong fluctuation regime (SFL) as:

$$
P_{\mathrm{w}}(l ; \theta, \alpha)=P_{\pi}(l ; \theta-\alpha) .
$$

This relationship is not restricted to the shallow limit $\alpha \rightarrow 0$ but it is also observed for acute wedges [23] and it has been confirmed by Ising model exact calculations [24] and computer simulations [25]. As a consequence, the critical exponents which characterize both transitions must be the same and the different moments and related quantities of the interfacial height PDF at the wedge mid-point (as $l_{\mathrm{w}}, \xi_{\perp}$, etc.) fullfil covariance relationships (i.e. $l_{\mathrm{w}}(\theta, \alpha)=l_{\pi}(\theta-\alpha)$ and so on). Furthermore, wedge covariance leads to some new results for wetting [14]. For example, it predicts that for thermal disorder the wetting wandering exponent $\zeta$ must be $\zeta=1 / 2[14]$. On the other hand, the relation between the wedge excess free energy and the point tension $\tau(\theta)$ implies that:

$$
l_{\pi}(\theta)=-\frac{\tau^{\prime}(\theta)}{2 \Sigma}
$$

This expression leads to the Indekeu-Robledo conjecture [26], which is consistent with exact Ising model calculations [16].

\section{Classical wedge covariance}

Now we turn to 3D systems with short-ranged forces. For this case, the binding potential reflects the decay of the local microscopic order parameter (density, magnetization) rather than the real intermolecular interactions. The binding potential at bulk coexistence is usually taken to have the form:

$$
W(l)=-a \mathrm{e}^{-\kappa l}+b \mathrm{e}^{-2 \kappa l},
$$

where $\kappa$ is the inverse bulk correlation length of the wetting phase. The leading order term vanishes at the mean-field wetting temperature $T_{\mathrm{w}}^{\mathrm{MF}}$ as $a \propto\left(T_{\mathrm{w}}^{\mathrm{MF}}-T\right)$, whilst $b$ is usually taken to be a positive constant at $T_{\mathrm{w}}^{\mathrm{MF}}$. Formally, eq. (16) of the binding potential can be inferred from a constrained fluctuation sum as in the Fisher-Jin approach which also leads to the presence of a position-dependent stiffness coefficient [17]. However, in the present analysis we will neglect such a position-dependence in the stiffness.

At mean-field level the equilibrium thickness $l_{\pi}$ of the interface is obtained by minimization of $W(l)$ whilst the contact angle $\theta$ can be identified from $\Sigma \theta^{2} / 2=$ $-W\left(l_{\pi}\right)$. Thus for systems with short-ranged forces the mean-field results are

$$
\kappa l_{\pi}=\ln 2 b / a=-\ln \sqrt{\frac{\Sigma}{2 b}} \theta,
$$

where we have used $a=\sqrt{2 \Sigma b} \theta$. From this expression we obtain that the critical exponents are $\beta_{\mathrm{s}}=0(\ln )$ and $\alpha_{\mathrm{s}}=0$. 
For shallow wedges, as mentioned before, the natural generalization of the planar model is the effective Hamiltonian (7). In the mean-field approach, the Hamiltonian is minimized subject to the appropriate boundary condition $l \rightarrow l_{\pi}+\alpha|x|$ as $|x| \rightarrow \infty$ and yields the Euler-Lagrange equation

$$
\Sigma l^{\prime \prime}(x)=W^{\prime}(l-\alpha|x|) .
$$

By integration, the 'energy' equation is obtained as:

$$
\frac{\Sigma}{2}\left(\left|l^{\prime}(x)\right|-\alpha\right)^{2}=\Delta W(l-\alpha|x|),
$$

where $\Delta W(l)=W(l)-W\left(l_{\pi}\right)$. At the wedge mid-point local height of the filling film verifies

$$
\frac{\Sigma}{2} \alpha^{2}=\Delta W\left(l_{\mathrm{w}}\right)
$$

This equation shows that for quite arbitrary choices of binding potential the wedge undergoes a filling transition when $\theta(T)=\alpha$, in agreement with thermodynamic arguments [9]. For the binding potential (16), the mid-point height is then given by

$$
\kappa l_{\mathrm{w}}(\theta, \alpha)=-\ln \sqrt{\frac{\Sigma}{2 b}}(\theta-\alpha) .
$$

This not only identifies the logarithmic divergence of the film thickness at the filling transition, $\beta_{\mathrm{f}}=0(\ln )$, but also reveals that the mean-field theory shows a classical analogue of wedge covariance observed in the $2 \mathrm{D}$ calculations:

$$
l_{\mathrm{w}}(\theta, \alpha)=l_{\pi}(\theta-\alpha)
$$

Classical wedge covariance is also manifest in other quantities like the whole equilibrium profile and the Gaussian fluctuations around the mean-field solutions [19].

For more general binding potentials, we must note that classical covariance is not a general feature of the mean-field theory of filling and critical wetting. For example, if we take the binding potential to have the form:

$$
W(l)=-a l^{-p}+b l^{-q}
$$

the filling and critical wetting exponents are distinct:

$$
\beta_{\mathrm{w}}=\frac{1}{p}, \quad \beta_{\mathrm{s}}=\frac{1}{q-p} .
$$

This automatically rules out the possibility of classical wedge covariance for binding potentials of the form (23). Nevertheless the results presented above showing wedge covariance for short-ranged forces do generalize to the class of potentials

$$
W(l)=-a \omega(l)+b \omega(l)^{2},
$$


where $\omega(l)$ corresponds to an arbitrary choice of monotonically decaying functions. Choosing $\omega=\mathrm{e}^{-\kappa l}$ we obtain the usual short-ranged binding potential whilst setting $\omega=l^{-p}$ one obtains a binding potential describing a particular type of multicritical wetting transition with $q=2 p$. Classical wedge covariance is obeyed by these systems, so we refer to the class of potentials (25) as classical wedge covariant binding potentials. Of course, only for the short-ranged case $\omega=\mathrm{e}^{-\kappa l}$ do we anticipate that such potentials have any physical significance. Nevertheless they do point to an important feature of the present analysis. It is easy to demonstrate that the wedge covariant potentials (25) all describe planar critical wetting transitions with vanishing specific heat exponent $\alpha_{\mathrm{s}}=0$. This situation is exactly the same as the fluctuation-induced non-classical covariance since for $\zeta \geq 1 / 2$ the value of the specific heat exponent in the SFL regime is $\alpha_{\mathrm{s}}=0$. This covers both cases, $\zeta=1 / 2$ and $\zeta=2 / 3$ in $2 \mathrm{D}$, where non-classical covariance is known to occur and it is tempting to speculate that the vanishing of the specific heat exponent either at mean-field level or beyond plays a key role for classical and non-classical covariance respectively.

Now we wonder if these results remain valid for acute wedges. As mentioned in the previous section, the RDN model (eq. (6)) seems to be the natural generalization of the Hamiltonian (eq. (7)) for acute wedges. So we have checked if the classical covariance is fulfilled. The mean-field equation for $l_{\mathrm{w}}$ in this case obeys

$$
\Sigma(\cos \theta-\cos \alpha)=W\left(l_{\mathrm{w}} \cos \alpha\right) .
$$

This equation predicts the correct filling transition boundary $\theta=\alpha$. If now we consider the short-ranged binding potential (eq. (16)), the asymptotic divergence of $l_{\mathrm{w}}$ is given by

$$
\kappa l_{\mathrm{w}} \sim-\sec \alpha \ln (\theta-\alpha) .
$$

So, the classical covariance result (eq. (22)) is not obeyed by the RDN model. Then it is natural to ask if classical wedge covariance is an artifact of the shallow wedge limit or that the RDN model is not a proper generalization of eq. (7). In order to answer this question, we turn our attention to studies of filling based on a microscopic Landau-like density functional theory. We remark that any effective interfacial Hamiltonian result must be consistent with results obtained from a more microscopic approach. For our Landau theory study we resort to a magnetic terminology rather than the fluids-based one considered earlier. At mean-field level the equilibrium order parameter $m(\mathbf{r})$ is translationally invariant along the wedge and so we can restrict ourselves to magnetization profiles in a two-dimensional space $\mathbf{r}=(x, z)$ with $x$ the coordinate across the wedge. The free-energy functional for the infinite wedge that we wish to minimize is

$$
F[m]=\int_{V} \mathrm{~d} \mathbf{r}\left\{\frac{1}{2}(\nabla m)^{2}-\frac{t}{2} m^{2}+\frac{u}{4} m^{4}-h m\right\},
$$

where the volume of integration is restricted to $z \geq \tan \alpha|x|$ for every $x$. The parameter $t$ measures the deviation from the bulk critical temperature (which is always finite) whilst $u>0$ for stability. The bulk field $h=0^{-}$so that the bulk magnetization is negative. The temperature dependence of the equilibrium profiles can be eliminated by measuring the magnetization in units of the bulk 
spontaneous magnetization $m_{0}(t)=\sqrt{t / u}$ and need not be specified further. Rather than using a local surface field $h_{1}$ and enhancement parameter $c$ we use fixed boundary conditions which set the surface magnetization to a positive value $m(x, \tan \alpha|x|)=m_{1}$ for all $x$. This is equivalent to $h_{1} \rightarrow \infty, c \rightarrow \infty$ limit with $m_{1}=h_{1} / c$ fixed in the model of Nakanishi and Fisher [27]. This choice ensures that the wetting transition pertinent to the planar wall-down spin interface is always second order. In the planar limit $\alpha=0$ the model can be solved analytically and exhibits a critical wetting transition when the surface magnetization

$$
m_{1}^{\text {wet }}=m_{0}
$$

which allows us to induce wetting (and filling in the wedge) by either increasing $m_{1}$ at fixed $t$ or varying $t$ at fixed $m_{1}$. We have chosen to vary $m_{1}$ at fixed temperature since this keeps the bulk magnetization and correlation length $\xi_{\mathrm{b}}=1 / \kappa=(2 t)^{-1 / 2}$ fixed. The contact angle within this model can be calculated analytically as

$$
\cos \theta=\frac{3 m_{1}}{2 m_{0}}\left(1-\frac{m_{1}^{2}}{3 m_{0}^{2}}\right)
$$

so that near the wetting transition $\theta \propto\left(m_{0}-m_{1}\right) / m_{0}$ where $\left(m_{0}-m_{1}\right) / m_{0}$ may be regarded as the temperature-like linear scaling field. We also remark that near the filling transition the scaling field $\theta-\alpha$ is equivalent to $\left(m_{1}^{\text {fill }}-m_{1}\right) / m_{0}$.

We have numerically minimized a discretized version of the continuum Landau free-energy functional. Details on the numerical procedures have been presented elsewhere [19]. The results of our study are presented in figure 2. The numerically

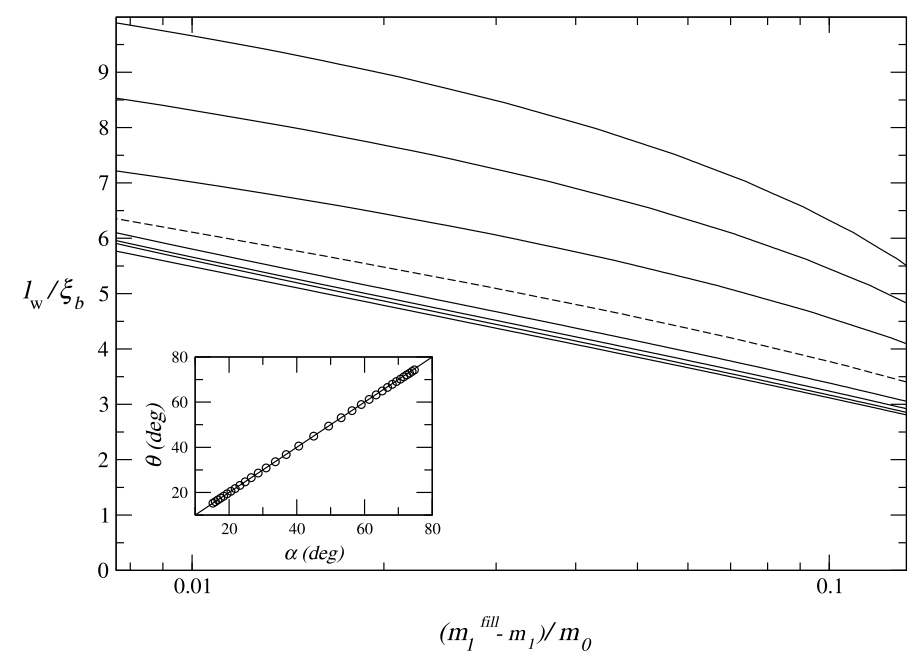

Figure 2. Plot of the reduced mid-point interface height, $l_{\mathrm{w}} / \xi_{\mathrm{b}}$, against the reduced surface magnetization, $m_{1} / m_{0}$, for a range of $\alpha$ between approximately $15^{\circ}$ (bottom) and $75^{\circ}$ (top). The dashed line corresponds to $\alpha=45^{\circ}$. Inset: Plot of the contact angle at the filling transition, $\theta$, vs. the wedge angle $\alpha$. The continuous line corresponds to the theoretical prediction (eq. (3)). 
determined phase boundary for tilt angles between $15^{\circ}$ and $75^{\circ}$ is in excellent agreement with the theoretical prediction $\theta=\alpha$. We have also studied the meniscus profile defined as the surface of isomagnetization $m=0$. For wedges with tilt angles $\alpha \leq \pi / 4$ there is clear evidence for the logarithmic growth of the mid-point filling height

$$
\kappa l_{\mathrm{w}}=A \ln \left(m_{1}^{\text {fill }}-m_{1}\right)+C
$$

with a universal, angle independent constant $A=1.02 \pm 0.04$ consistent with the classical covariance result $A=1$ and in disagreement with the RDN model predictions. The constant $C$ is non-universal as is the size of the asymptotic critical regime which decreases with increasing wedge angle. Nevertheless, even for the most acute angles we consider, there is no evidence that the amplitude $A$ differs from unity. Consequently, classical wedge covariance is not an artifact but a general property which must be fulfilled by any physically acceptable interfacial model. The failure of the RDN model to account for classical wedge covariance in more acute wedges is a surprising result. Indeed the RDN model does predict non-classical wedge covariance for 2D systems with strictly short-ranged forces [16]. However, this is not unexpected since non-classical wedge covariance is a fluctuation-induced phenomenon for which the precise form of the binding potential (determining the way in which short-range forces are modelled) is irrelevant. This contrasts with classical wedge covariance which reflects the precise form of the underlying interfacial Hamiltonian model. Clearly the assumption that the wall-interface interaction occurs via the normal distance to points on the closest wall is incorrect. The absence of a $\sec \alpha$ prefactor in the Landau numerics indicate that the correct measure of this is more akin to an effective local, vertical interaction similar to the shallow wedge model. Now we confront the problem of constructing an interfacial model for $3 \mathrm{D}$ short-ranged forces which is consistent with well-established versions for planar substrates and also verifies classical wedge covariance for shallow and acute wedges.

\section{The non-local model}

Consider a Landau-Ginzburg-Wilson Hamiltonian based on a continuum orderparameter (magnetization) $m(\mathbf{r})$ in a semi-infinite geometry with bounding surface described by a single-valued height function $\psi(\mathbf{x})$ where $\mathbf{x}=(x, y)$ is the parallel displacement vector. Denoting the surface magnetization by $m_{1}(\mathbf{x})$ we write

$$
H_{\mathrm{LGW}}=F[m]+\int \mathrm{d} s_{\psi} \phi_{1}\left(m_{1}\right),
$$

where $F[m]$ is given by eq. (28) where the volume $V$ is the upper semi-space $z>0$, $\mathrm{d} s_{\psi}=\sqrt{1+(\nabla \psi)^{2}} \mathrm{~d} \mathbf{x}$ is the wall area element and $\phi_{1}\left(m_{1}\right)$ is a suitable surface potential [27]. Following FJ [17] we identify the interfacial model $H=H_{\mathrm{LGW}}\left[m_{\Xi}(\mathbf{r})\right]$ where $m_{\Xi}(\mathbf{r})$ is the profile which minimizes eq. (32) subject to a given interfacial configuration. FJ determined $m_{\Xi}(\mathbf{r})$ perturbatively in terms of local planar constrained profiles [17]. Here we construct $m_{\Xi}$ non-perturbatively using Greens' functions or equivalently correlation functions defined within the constrained wetting layer. The latter reduces to the classical $\mathrm{OZ}$ form over relevant distances 
provided the wetting layer is much thicker than its bulk correlation length $\kappa^{-1}$. This non-local (NL) Hamiltonian can be written as

$$
H=\int \mathrm{d} \mathbf{x}\left\{\Sigma \sqrt{1+(\nabla l)^{2}}+h(l-\psi)\right\}+W[l, \psi],
$$

where $h$ is proportional to the bulk field. There is no explicit position-dependent tension but rather a binding potential functional with three leading contributions

$$
W[l, \psi]=-a \Omega_{1}^{1}[l, \psi]+b_{1} \Omega_{1}^{2}[l, \psi]+b_{2} \Omega_{2}^{1}[l, \psi],
$$

where $a, b_{1}$ and $b_{2}$ are best regarded as phenomenological parameters to be identified later. Each $\Omega_{\mu}^{\nu}$ represents integrated two-point interactions between $\mu$ and $\nu$ points on the wall and interface mediated by the (rescaled) bulk OZ correlation function $K(r)=\kappa \mathrm{e}^{-\kappa r} / 2 \pi r$. These can be viewed as contributions to the free energy of a constrained thin film arising from tube-like fluctuations of the bulk phase which tunnel from the interface to the wall [16]. The first term involves only one tube

$$
\Omega_{1}^{1}[l, \psi]=\int \mathrm{d} s_{\psi}^{1} \int \mathrm{d} s_{l}^{2} K\left(r_{12}\right)
$$

where $\mathrm{d} s_{l}^{\mu}=\sqrt{1+\left(\nabla l\left(\mathbf{x}_{\mu}\right)^{2}\right.} \mathrm{d} \mathbf{x}_{\mu}$ etc. and $r_{12}=\sqrt{\left|\mathbf{x}_{12}\right|^{2}+\left(\psi\left(\mathbf{x}_{1}\right)-l\left(\mathbf{x}_{2}\right)\right)^{2}}$ is the distance between two points on the interface and wall. The last two terms

$$
\Omega_{1}^{2}[l, \psi]=\int \mathrm{d} s_{\psi}^{1}\left\{\int \mathrm{d} s_{l}^{2} K\left(r_{12}\right)\right\}^{2}, \quad \Omega_{2}^{1}[l, \psi]=\Omega_{1}^{2}[\psi, l]
$$

involve two tubes and may be viewed as a self-interaction between points on the same interface or wall induced by the presence of a second surface. Figure 3 shows a diagrammatic representation of such terms. The upper and lower lines represent typical non-planar configurations of the interface and wall, the undulated line joining them represents the interaction function $K\left(r_{12}\right)$ whilst the solid dots imply integration over the area of each surface.

For general wall and interfacial configurations all contributions to $W[l, \psi]$ are NL. However, if both the wall and interface are flat, $\psi(\mathbf{x})=0, l(\mathbf{x})=l$, the

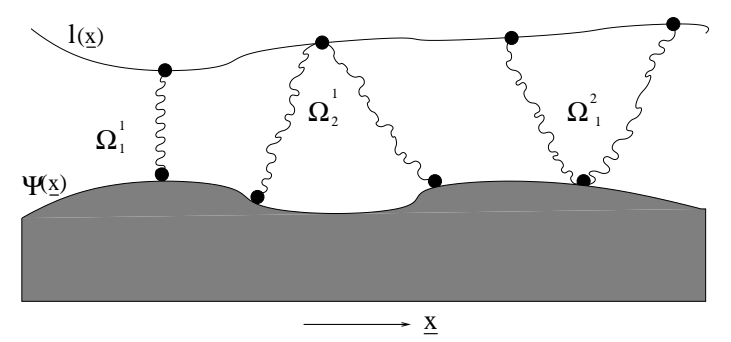

Figure 3. Schematic illustration of the diagrams which represent the leading order contributions to $W[l, \psi]$. 
Hamiltonian per unit area $W(l)=W[l, 0] / A$ reduces to the standard form of the binding potential appearing in local models (eq. (16)) with $b \equiv b_{1}+b_{2}$. For the more general case of a non-planar interface near a planar wall, two contributions to the binding potential functional are local since

$$
\Omega_{\mu}^{1}[l, 0]=\int \mathrm{d} s_{l}^{1} \mathrm{e}^{-\mu \kappa l\left(\mathbf{x}_{1}\right)}, \quad \mu=1,2 .
$$

However $\Omega_{1}^{2}$ remains NL and can be rewritten as a two-body repulsive interaction

$$
\Omega_{1}^{2}[l, 0]=\iint \mathrm{d} s_{l}^{1} \mathrm{~d} s_{l}^{2} S\left(\left|\mathbf{x}_{12}\right| ; \bar{l}\right)
$$

where $\bar{l}=\left[l\left(\mathbf{x}_{1}\right)+l\left(\mathbf{x}_{2}\right)\right] / 2$ and

$$
S(x ; \bar{l})=\frac{\kappa^{2}}{2 \pi} \int_{2 \kappa \bar{l}}^{\infty} \mathrm{d} t \frac{\mathrm{e}^{-\sqrt{t^{2}+\kappa^{2} x^{2}}}}{\sqrt{t^{2}+\kappa^{2} x^{2}}} \approx \frac{\kappa}{4 \pi \bar{l}} \mathrm{e}^{-2 \kappa \bar{l}-\kappa x^{2} / 4 \bar{l}}
$$

valid for $\kappa \bar{l} \gg 1$. In the small gradient limit, $|\nabla l| \ll 1$, the NL term can be expanded and the model reduces to

$$
H[l, 0] \approx \int \mathrm{d} \mathbf{x}\left\{\frac{\Sigma(l)}{2}(\nabla l)^{2}+W(l)\right\}
$$

with stiffness coefficient

$$
\Sigma(l)=\Sigma-a \mathrm{e}^{-\kappa l}-2 b_{1} \kappa l \mathrm{e}^{-2 \kappa l}+\cdots
$$

precisely recovering the FJ model and uniquely identifying $a, b_{1}$ and $b_{2}$. In particular $a$ measures the deviation from the MF critical wetting temperature, $b_{2} \propto a^{2}$ and the sign of $b_{1}$ determines the order of the MF transition. Thus the origin of the $\kappa l \mathrm{e}^{-2 \kappa l}$ contribution, crucial in the FJ analysis, can be traced directly to a perturbative treatment of the NL contribution $\Omega_{1}^{2}$.

Now consider fluid adsorption in a wedge geometry $(\psi=\tan \alpha|x|)$. The NL model satisfies the necessary requirement of classical wedge covariance (eq. (22)). The reason for this can be traced to the structure of the NL binding potential. Since filling precedes wetting $(a \neq 0)$, the dominant term is $\Omega_{1}^{1}$. Now for a flat interfacial configuration $l(\mathbf{x})=l_{0}$, near a non-planar wall both $\Omega_{1}^{1}$ and $\Omega_{1}^{2}$ are local with, for example

$$
\left.\Omega[l, \psi]\right|_{l=l_{0}}=\int \mathrm{d} \mathbf{x} \sqrt{1+(\nabla \psi)^{2}} \mathrm{e}^{-\kappa\left(l_{0}-\psi(\mathbf{x})\right)}
$$

showing that the effective local interaction occurs via the vertical distance to the surface. Near the filling phase boundary the interface is essentially flat in the filled section of the wedge and the $\Omega_{1}^{1}$ contribution must be of the above form. This is sufficient to ensure covariance. We also remark that for wetting at more general non-planar walls the NL model reproduces the precise form of the stiffness matrix appearing in approximate two-field models [28] valid for $|\nabla l| \ll 1$ and $|\nabla \psi| \ll 1$. 
This means that in application to complete wetting the NL theory satisfies exact sum rules [29].

Our NL model shows unexpected behaviour when applied to the critical wetting at a planar substrate. The standard CW model famously predicts non-universal criticality dependent on the wetting parameter $\omega=k_{\mathrm{B}} T \kappa^{2} / 4 \pi \Sigma[30]$. However, this strongly disagrees with Ising model simulation studies [31] which show only minor deviations from MF-like critical wetting behaviour (for the experiments, see ref. [32]). The more refined FJ model provides a possible explanation of this discrepancy since the $\Sigma(l)$ term drives the transition first order for physical values of $\omega[18]$. Here we show that the stiffness instability is not a robust mechanism since the wetting transition described by the NL model remains continuous. A linear RG theory can be constructed provided we first expand $\sqrt{1+(\nabla l)^{2}}$ to square gradient order. The local terms $\Omega_{\mu}^{1}$ generate effective binding potential and positiondependent stiffness contributions which renormalize as in refs $[18,30]$. We focus on the renormalization of the NL potential $S(x ; \bar{l})$ which controls the order of the phase transition since it is responsible for the $-l \mathrm{e}^{-2 \kappa l}$ term in the perturbative $|\nabla l| \ll 1$ limit. After renormalizing up to a scale $b=\mathrm{e}^{t}$ the NL term $\Omega_{1}^{2}$ retains its two-body form but with a modified potential $S_{t}(x ; \bar{l})$ satisfying the flow equation:

$$
\frac{\partial S_{t}}{\partial t}=4 S_{t}+x \frac{\partial S_{t}}{\partial x}+\omega \kappa^{-2}\left(\frac{1+J_{0}(\Lambda x)}{2}\right) \frac{\partial^{2} S_{t}}{\partial \bar{l}^{2}},
$$

where $J_{0}(x)$ is a Bessel function of the first kind and $\Lambda$ is the momentum cut-off. This equation has the formal solution:

$$
S_{t}(x ; \bar{l})=\mathrm{e}^{4 t} \int_{-\infty}^{\infty} \mathrm{d} l \frac{\kappa S_{0}\left(x \mathrm{e}^{t}, l\right) \exp \left(\frac{-(\kappa l-\kappa \bar{l})^{2}}{4 \omega \Phi\left(\Lambda x \mathrm{e}^{t}, \Lambda x\right)}\right)}{\sqrt{4 \pi \omega \Phi\left(\Lambda x \mathrm{e}^{t}, \Lambda x\right)}},
$$

where $\Phi(a, b)=\int_{b}^{a} \mathrm{~d} t\left[1+J_{0}(t)\right] / 2 t$. We choose $S_{0}(x, l)=\Theta(l) S(x, l)$, with $\Theta(l)$ the Heaviside step function and $S(x, l)$ given by eq. (39). As $t \rightarrow \infty, S_{t}(x, l)$ becomes increasingly localized around $x=0$. Using a matching technique we renormalize to a scale $\mathrm{e}^{t^{*}}$ at which the curvature of the effective binding potential $W_{t}(l)$ at its global minimum is of order of $\Sigma \kappa^{2}$. Our $W_{t}(l)$ has a local contribution due to the $\Omega_{1}^{1}$ and $\Omega_{2}^{1}$ processes, and a NL contribution which is obtained from the expansion of $\Omega_{1}^{2}$ in powers of $\nabla l$ :

$$
W_{t}^{\mathrm{NL}}(l)=2 \pi \int_{0}^{\infty} \mathrm{d} x x S_{t}(x, l) .
$$

Numerical integration of the RG flow equation shows that the wetting transition is always second order, and quantitatively similar to the non-universality exhibited by the CW model. This fact can be rationalized by noting that $\Phi\left(\Lambda x \mathrm{e}^{t}, \Lambda x\right) \sim t$ as $t \rightarrow \infty$ and $\kappa x \lesssim \mathrm{e}^{-t}$, which is the range where $S_{0}\left(x \mathrm{e}^{t}, l\right)$ is non-negligible. Consequently, in our NL model there is no stiffness instability. The difference with the RG predictions of the FJ model arises specifically from non-locality. Mathematically the FJ flow equations can be recovered from eq. (43) if we approximate the Bessel function term by its quadratic expansion in $x$. However this is not valid at large distances and invalidates the stiffness instability. 
In order to check the RG predictions, we have performed Monte Carlo simulations of the CW, FJ and NL Hamiltonians (with the approximation $\sqrt{1+(\nabla l)^{2}} \approx 1+$ $\left.(\nabla l)^{2} / 2\right)$. Following ref. [33] we discretize by introducing a $L \times L$ lattice of spacing $\sigma$ with periodic boundary conditions in the directions parallel to the surface, but treating the interfacial position height as continuous variables. We chose $\sigma=$ $3.1623 \kappa^{-1}$ so that $\Lambda \kappa^{-1} \sim \pi / \kappa \sigma \lesssim 1$, and also set $\omega=0.8$ and $b_{1}=2.5 \kappa^{2} k_{\mathrm{B}} T$ which are reasonable Ising-like parameters. We anticipate the critical wetting phase boundary remains $\mathrm{MF}(a=0)$ for the CW and NL theories [30], whilst the FJ exhibits a first-order transition at higher temperatures [18]. Figure 4 describes the behaviour of the mean wetting layer thickness $\langle l\rangle$ and the surface magnetizationlike operator $\Delta m_{1}=\left\langle\mathrm{e}^{-\kappa l}\right\rangle$ along the MF critical wetting isotherm $a=0, h \rightarrow 0$. The FJ model clearly describes partial wetting in this limit which is consistent with a fluctuation-induced first-order transition. On the other hand, the CW and NL models are qualitatively similar, showing continuous wetting. The divergence of the film thickness is well-described by the RG result $\kappa\langle l\rangle \sim-\sqrt{2 \omega} \ln h$ even for

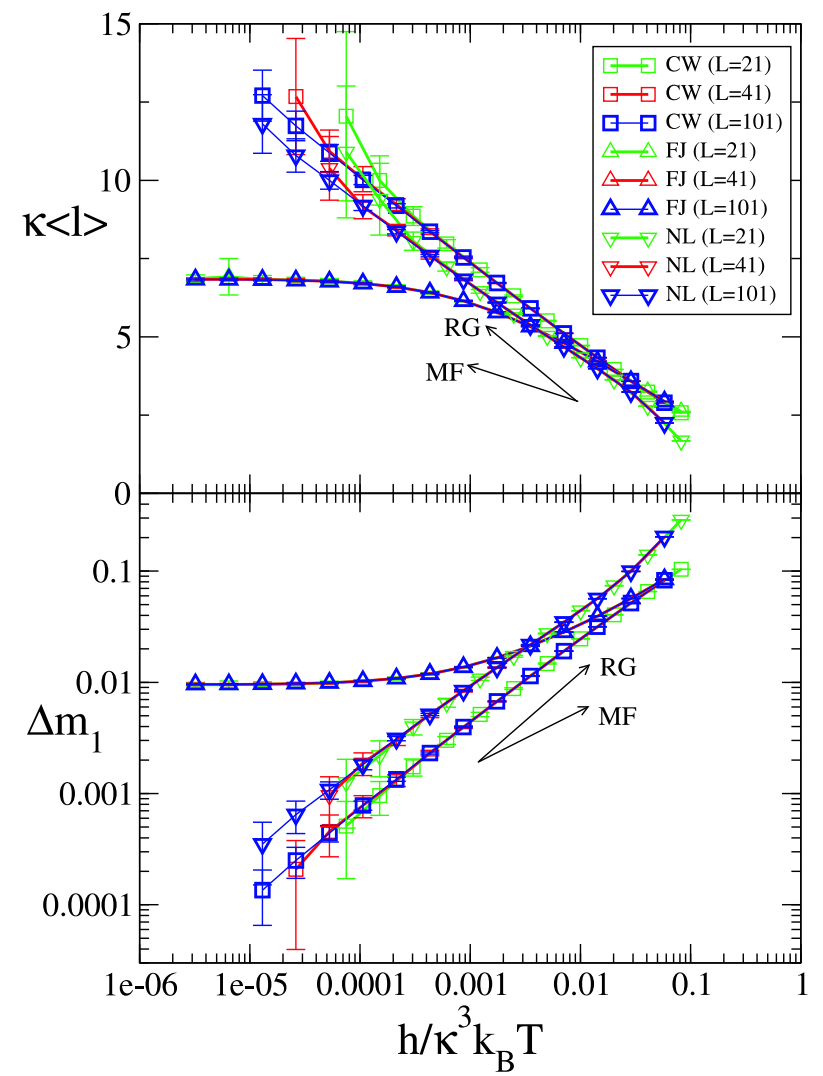

Figure 4. Plot of the mean wetting layer $\langle l\rangle$ and surface magnetization operator $\Delta m_{1}$ vs. $h$ obtained by computer simulations of the CW, FJ and our NL model for $\omega=0.8, a=b_{2}=0$ and $b_{1} / \kappa^{2} k_{\mathrm{B}} T=2.5$. 
moderately thick wetting layers. However, the surface magnetization shows a much larger pre-asymptotic critical regime. The asymptotic non-universal behaviour $\Delta m_{1} \sim h^{1-1 / 2 \nu_{\|}}$, with $\nu_{\|}=(\sqrt{2}-\sqrt{\omega})^{-2}$ is not observed until the wetting layer $\kappa\langle l\rangle \sim 10$ for very large lattice sizes $(\kappa L \sim 300)$. This is strongly suggesting that current Ising model simulations will not be able to observe significant deviation from MF behaviour provided they focus on surface quantities.

\section{Conclusions}

In this paper we review some recent results regarding adsorption in the wedge geometry. We show the existence of a classical wedge covariance relation between the filling transition and the wetting transition for short-ranged forces, which becomes a stringent test for effective interfacial models at the mesoscopic scale. We construct a non-local model which fulfils this condition and we anticipate that it may be applied to more complex geometries such as adsorption in parabolic grooves or around spherical or cylindrical objects. When applied to wetting of planar substrates, we find that fluctuations does not drive the transition first-order (even when the model presents an effective position-dependent stiffness term as in the FJ model).

\section{Acknowledgments}

The authors gratefully acknowledge M J Greenall, A Lazarides, J Gibbons, A J Wood and D B Abraham for discussions and contributions to this work. JMR-E acknowledges partial financial support from Secretaría de Estado de Educación y Universidades (Spain), co-financed by the European Social Fund, and from the European Commission under Contract MEIF-CT-2003-501042.

\section{References}

[1] S Herminghaus et al, Adv. Mater. 11, 1393 (1999) F R Service, Science 282, 399 (1998)

[2] A Terry et al, Science 296, 1841 (2002)

G M Whitesides and A D Strock, Phys. Today 54, 42 (2001)

[3] H Gau et al, Science 283, 46 (1999)

[4] C Rascón and A O Parry, Nature (London) 407, 986 (2000)

[5] L Bruschi, A Carlin and G Mistura, Phys. Rev. Lett. 89, 166101 (2002)

[6] P Concus and R Finn, Proc. Natl. Acad. Sci. USA 63, 292 (1969)

[7] Y Pomeau, J. Colloid Interface Sci. 113, 5 (1986)

[8] E H Hauge, Phys. Rev. A46, 4994 (1992)

[9] K Rejmer, S Dietrich and M Napiórkowski, Phys. Rev. E60, 4027 (1999)

[10] A O Parry, C Rascón and A J Wood, Phys. Rev. Lett. 85, 345 (2000)

[11] A O Parry, A J Wood and C Rascón, J. Phys.: Condens. Matter 13, 4591 (2001)

[12] A O Parry, C Rascón and A J Wood, Phys. Rev. Lett. 83, 5535 (1999)

[13] A O Parry, A J Wood and C Rascón, J. Phys.: Condens. Matter 12, 7671 (2000)

[14] A O Parry, M J Greenall and A J Wood, J. Phys.: Condens. Matter 14, 1169 (2002) 
[15] See for example, R Evans, in: Fundamentals of inhomogeneous fluids edited by D Henderson (Dekker, New York, 1992)

[16] D B Abraham, J T Chayes and L Chayes, Commun. Math. Phys. 96, 439 (1984)

[17] M E Fisher and A J Jin, Phys. Rev. B44, 1430 (1991) A J Jin and M E Fisher, Phys. Rev. B47, 7365 (1993)

[18] M E Fisher and A J Jin, Phys. Rev. Lett. 69, 792 (1992) A J Jin and M E Fisher, Phys. Rev. B48, 2642 (1993) C J Boulter, Phys. Rev. Lett. 79, 1897 (1997)

[19] M J Greenall, A O Parry and J M Romero-Enrique, J. Phys.: Condens. Matter 16, 2515 (2004)

[20] A Milchev, M Müller, K Binder and D P Landau, Phys. Rev. Lett. 90, 136101 (2003)

[21] A Milchev, M Müller, K Binder and D P Landau, Phys. Rev. E68, 031601 (2003)

[22] J M Romero-Enrique, A O Parry and M J Greenall, Phys. Rev. E69, 061604 (2004)

[23] D B Abraham, A O Parry and A J Wood, Europhys. Lett. 60, 106 (2002)

[24] D B Abraham and A Maciolek, Phys. Rev. Lett. 89, 286101 (2002)

[25] E V Albano, A De Virgiliis, M Müller and K Binder, J. Phys.: Condens. Matter 15, $333(2003)$

[26] J O Indekeu and A Robledo, Phys. Rev. E47, 4607 (1993)

[27] H Nakanishi and M E Fisher, Phys. Rev. Lett. 49, 1565 (1982)

[28] C J Boulter and A O Parry, Phys. Rev. Lett. 74, 3403 (1995)

[29] See J R Henderson, in: Fundamentals of inhomogeneous fluids edited by D Henderson (Dekker, New York, 1992)

[30] E Brézin, B I Halperin and S Leibler, Phys. Rev. Lett. 50, 1387 (1983)

D S Fisher and D A Huse, Phys. Rev. B32, 247 (1985)

[31] K Binder, D P Landau and D M Kroll, Phys. Rev. Lett. 56, 2272 (1986)

[32] D Ross, D Bonn and J Meunier, Nature (London) 400, 737 (1999)

[33] G Gompper and D M Kroll, Phys. Rev. B37, 3821 (1988) 